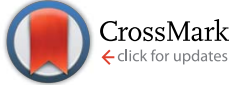

Cite this: RSC Adv., 2016, 6, 31919

Received 25th February 2016

Accepted 21st March 2016

DOI: $10.1039 / \mathrm{c} 6 \mathrm{ra05024g}$

www.rsc.org/advances

\section{Wavelength-tuneable laser emission from stretchable chiral nematic liquid crystal gels via in situ photopolymerization $\uparrow$}

\begin{abstract}
S. M. Wood, ${ }^{a}$ F. Castles, ${ }^{b}$ S. J. Elston ${ }^{a}$ and S. M. Morris ${ }^{* a}$
Stretchable liquid crystal laser gels are free-standing films with emission wavelengths that may be tuned by applying a mechanical strain. These laser gels offer potential for industrial applications such as microactuators and pressure sensors due to their small dimensions and suitability for solution processing. Here, we demonstrate examples of such laser gels that comprise multiple regions emitting at different wavelengths, each of which may be reversibly and selectively tuned without measurable hysteresis. These gels are created from a combination of reactive and non-reactive mesogenic molecules using an in situ photo-polymerization technique that is compatible with a variety of commercially available materials and is therefore more versatile than previous methodologies for creating elastomeric liquid crystal lasers. In this paper, we vary the concentration of reactive mesogen and study the corresponding change in the mechanochromic properties of the resulting films. By doping the gels with a fluorescent dye, laser emission that can tuned continuously (by $\sim 40 \mathrm{~nm}$ ) upon mechanical extension along a direction that is perpendicular to the helicoidal axis of the chiral nematic liquid crystal phase is observed. Moreover, tuning of the wavelength is found to be reversible and does not exhibit any measurable hysteresis, thereby allowing repeatable selection of a desired laser wavelength by controlling the film elongation. By virtue of the versatility of the technique, it is possible to photo-polymerise different areas of the thin-films at different temperatures to pattern the gels in such a way that different regions of the gel emit at different laser wavelengths.
\end{abstract}

\section{Introduction}

Band-edge liquid crystal (LC) lasers typically consist of a chiral mesophase, such as the chiral nematic, chiral smectic or blue phase, which is doped with a low concentration by weight of a fluorophore in the form of a high quantum yield fluorescent dye. ${ }^{1-4}$ Numerous reports have shown that these thin film $(\sim 10$ $\mu \mathrm{m}$ thick) lasers exhibit a range of desirable emission characteristics, such as narrow spectral linewidths and simultaneous multi-wavelength emission from a single liquid crystalline sample. ${ }^{5,6}$ In contrast to conventional inorganic semiconductor and rare-earth materials that are used in commercially available lasers, these materials are also compatible with a range of lowcost, high throughput manufacturing processes such as roll-toroll printing and drop-on-demand inkjet printing. ${ }^{7,8}$ Combined, these features demonstrate that LC lasers have potential in

${ }^{a}$ Department of Engineering Science, University of Oxford, Parks Road, Oxford, OX1 3PJ, UK. E-mail: stephen.morris@eng.ox.ac.uk

${ }^{b}$ Department of Materials Science, University of Oxford, Parks Road, Oxford, OX1 3PH, $U K$

$\uparrow$ Electronic supplementary information (ESI) available: One figure and one video. See DOI: 10.1039/c6ra05024g a broad variety of applications including display devices, sensors, and biomedical diagnostic tools.

The mechanism responsible for laser emission in these structures can be understood in terms of the large density of photon states, and consequently large photon dwell time, that occurs at the edge of the photonic band gap (PBG) of the chiral LC phase., ${ }^{9,10}$ This gap in the photonic bands arises due to the combination of a macroscopic helicoidal structure, which has a pitch on the order of the wavelength of light, and the birefringence of the LC phase. Importantly, this macroscopic structure self-assembles, which is in contrast to conventional distributed feedback structures whereby the grating has to be artificially written-in to the active gain medium.

Arguably, the most studied LC band-edge laser has been based upon the chiral nematic phase, which gives rise to a onedimensional PBG that reflects only the one handedness of circularly polarized light that matches the rotation sense of the helicoidal structure. To observe laser emission at the edge of the band-gap, it is often necessary to disperse a fluorophore into the chiral nematic LC (although laser emission from the LC host has also been previously observed ${ }^{11}$ ). For maximum coupling efficiency, it is important to match the wavelength of one of the band-edges to that of the peak of the fluorescence spectrum. In principle, laser emission can be observed at either band-edge, 
however, the lowest excitation threshold is usually observed for the mode at the long-wavelength band-edge due to the coalignment of the electric field vector of the resonant mode with the orientation of the transition dipole moment for emission. ${ }^{\mathbf{1 0}}$

A key advantage of using chiral LCs as band-edge lasers is that the spectral position of the PBG position can be influenced by a variety of external stimuli. To date, a vast array of methods that can be used to perform in situ wavelength-tuning of LC lasers has been reported. These include thermal tuning, ${ }^{12}$ tuning with electric ${ }^{13}$ and magnetic ${ }^{14}$ fields, photo-tuning using UV and visible light, ${ }^{15}$ and tuning by applying a mechanical strain. ${ }^{16-18}$ Mechanical tuning is of interest for potential applications where deformable photonic structures are required, such as pressure sensors and optical micro-actuators. ${ }^{16}$ On application of a lateral mechanic strain, the LC's helicoidal structure is contracted, leading to a shortening of the pitch and a blue-shift of the PBG. ${ }^{18,19}$

Mechanical wavelength tuning of a LC laser was first achieved in a so-called liquid single-crystal elastomer, ${ }^{16}$ prepared via the Kim and Finkelmann anisotropic deswelling method. ${ }^{20}$ However, as noted in ref. 20, the optical properties of such samples is far from perfect and, as a result, the laser performance is inferior to that of typical pristine chiral nematic samples in low molecular mass systems. In particular, the lasing threshold in the elastomer was three orders of magnitude larger than previous conventional systems. ${ }^{16,21}$ An alternative method was investigated by Schmidtke et al. whereby an uncrosslinked chiral nematic polymer was prepared between two glass substrates before being subsequently photo-crosslinked. ${ }^{21}$ This yielded a sharper PBG with a comparable tuning-range, however a highly aligned sample is again difficult to obtain via this method due to the large viscosity and disorder inherent in the initial polymer (for example, in ref. 20 the sample was annealed for two days to achieve the Grandjean texture).

The purpose of this study is to investigate a third fabrication methodology whereby a pristine chiral nematic sample is first formed in a monomeric system that incorporates reactive mesogens, before subsequent in situ photopolymerization and cross-linking. The in situ photopolymerization of LC polymers was pioneered by Broer, et al. ${ }^{22}$ and facilitates the creation of very highly aligned samples, typically in a matter of seconds or minutes, with only minimal degradation upon photopolymerization and cross-linking. While the efficacy of the method is limited to relatively thin samples (less than a few tens of $\mu \mathrm{m}$ thick), this is not a hindrance in the context of LC lasers, where the optimum LC layer thickness is $\sim 10 \mu \mathrm{m} .{ }^{23}$ This technique has already been successfully used to create elastomeric blue phase LC gels ${ }^{24}$ and (non-elastomeric) liquid crystal lasers. ${ }^{25}$ However, previously, lasers fabricated using this technique have not been made to be free-standing nor shown to be stretchable. Here, we dope the mixture of reactive and non-reactive mesogens with a fluorescent dye to create lasers whose output wavelength may be tuned reversibly and without hysteresis when a mechanical strain is applied. Furthermore, we demonstrate that, using the in situ photopolymerisation technique, it is possible to pattern regions with different pitches thereby resulting in multicolored laser emission from a single LC gel.
The paper is organised as follows: we first outline the procedure for fabricating the stretchable gels and then present results showing how the mechanochromic range and sensitivity of films containing different concentrations of the reactive species are affected when the films are subjected to a mechanical strain along a direction that is perpendicular to the helicoidal axis. Subsequently, we show wavelength tuneable laser emission from these gels and that the tuning appears to be reversible with minimal hysteresis. Finally, we show that by curing the devices at different temperatures, laser films that emit at multiple wavelengths can be fabricated, something which is not readily achievable using conventional chiral LC elastomers, and has not previously been demonstrated.

\section{Experimental}

\section{Mixture composition}

To form the base chiral nematic mixture consisting of nonreactive components, the high twisting power chiral dopant, R5011 (Merck), was dispersed into the achiral nematic LC, E7 (Merck) to yield a chiral nematic LC with a right-handed helicoid, as confirmed by polarising optical microscopy. Four samples consisting of different concentrations by weight of the reactive mesogen mixture are included in this study for comparison. The reactive mesogen mixture (UCL-011-K1, Dai Nippon Ink) consists of both mono- and di-acrylates, a photoinitiator and a thermal inhibitor. Details of the mixture compositions used here are provided in Table 1.

For the laser sample, the fluorescent dye PM597 (Exciton) was added to mixture $\mathrm{D}$ at a concentration of $1.0 \mathrm{wt} \%$. This dye was chosen because it is known to be soluble in nematic LCs, has a high quantum efficiency ( $~ 75 \%$ in E49 (ref. 26)) and does not absorb at UV wavelengths (which may adversely affect the UV-curing process and thus lead to non-uniform network morphologies).

\section{Sample preparation}

First of all, the mixtures were capillary filled into $20 \mu \mathrm{m}$-thick commercially available glass cells that consisted of anti-parallel rubbed polyimide alignment layers that were coated onto the inner substrates of the cell. To form a uniform standing helix (Grandjean) alignment, the samples were cooled from the isotropic phase and, if necessary, were subjected to mechanical shearing by applying pressure to the cell in order to give a good alignment, which can be seen from POM images and the PBGs in the transmission spectra. Following the alignment process, the

Table 1 Compositions of mixtures containing different concentrations of reactive mesogen mix

\begin{tabular}{llll}
\hline Mixture & E7 $[\mathrm{wt} \%]$ & R5011 $[\mathrm{wt} \%]$ & UCL-011-K1 [wt\%] \\
\hline A & 67.3 & 2.7 & 30.0 \\
B & 57.4 & 2.6 & 40.0 \\
C & 48.6 & 2.6 & 48.8 \\
D & 39.9 & 2.4 & 57.7
\end{tabular}




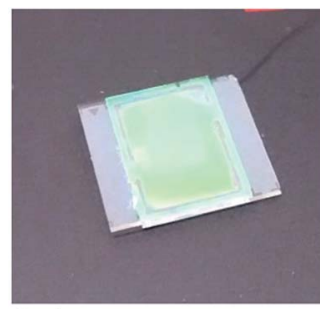

(a)

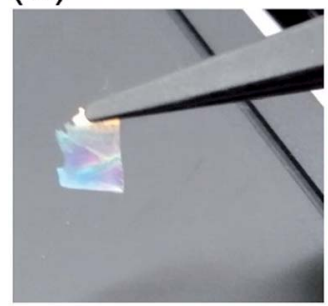

(c)

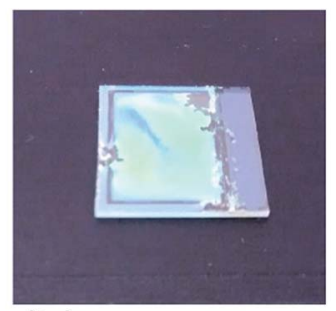

(b)

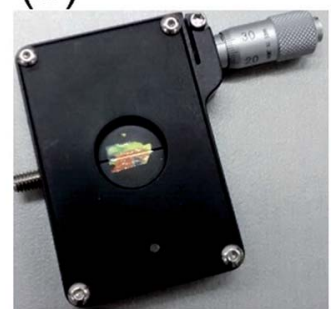

(d)
Fig. 1 Process for creating the stretchable films. (a) The mixture is aligned in a glass cell (20 $\mu \mathrm{m}$ spacing) and photo-cured; (b) the cell is peeled apart with a scalpel; (c) the film is delaminated using a scalpel; (d) the film is placed across a variable aperture and elongated.

samples were then illuminated at a wavelength of $\lambda=365 \mathrm{~nm}$ and an output power density of $185 \mathrm{~mW} \mathrm{~cm} \mathrm{~cm}^{-2}$ at the light source, which was held at a distance of $20 \mathrm{~cm}$ away from the sample. To ensure uniform curing conditions, each cell was illuminated for $120 \mathrm{~s}$ from one side of the cell and then the other side using a UVcuring system (Thorlabs CS2010). Fig. 1a shows a film that has been photo-cured between glass substrates. Afterwards, the glass cells were separated, as shown in Fig. 1b, and the films were then delaminated using a scalpel to yield the free-standing films of the type shown in Fig. 1c - with average dimensions $\sim 5 \mathrm{~mm} \times$ $10 \mathrm{~mm}$.

To create multi-colored samples, one half of the cell was masked to shield it from illumination while the other half was photo-cured. The uncured region was then unmasked and the sample was heated to $45^{\circ} \mathrm{C}$ using a Linkam TP93 hot stage and controller. The increase in temperature results in a shortened pitch in the half that has not been cured. Photo-curing at an elevated temperature locks-in a structure with a shorter pitch and hence a band-gap occurring at shorter wavelengths.

\section{Spectral analysis measurements}

The films were mounted on a variable-width aperture (Thorlabs VA100/M) that was comprised of two blades that could be opened between $25 \mu \mathrm{m}$ and $6 \mathrm{~mm}$ using a micrometric screw, as shown in Fig. 1d with a free-standing gel mounted on the aperture. The samples were then illuminated by a halogen white light source (Ocean Optics HL-2000-FHSA) focussed to a spot size of $\sim 500 \mu \mathrm{m}$ diameter at the aperture using a $20 \times, 0.5 \mathrm{NA}$ objective. The transmission spectra were recorded on a fibercoupled spectrometer (Ocean Optics USB2000+ UV-VIS; resolution $1.7 \mathrm{~nm}$ ) with a $600 \mu \mathrm{m}$-diameter fibre. To check the alignment and observe the change in the optical texture upon the application of a uniaxial mechanical strain, we used an
Olympus BX51 optical polarizing microscope fitted with a digital SLR camera (Nikon D90) in the photo-tube.

\section{Laser emission studies}

The samples were optically pumped with a frequency-doubled Nd:YAG laser (CryLas 6FTSS355-Q4-S) emitting 1 ns pulses at $532 \mathrm{~nm}$ with a repetition rate of $10 \mathrm{~Hz}$. The pump laser was focused onto the devices to a spot size of $\sim 200 \mu \mathrm{m}$ diameter and the output was measured perpendicular to the films along the direction of the helicoidal axis. A $550 \mathrm{~nm}$ long pass filter was used to block the pump beam from being detected by the spectrometer. Emission was collected using a microscope objective $(5 \times, 0.25 \mathrm{NA})$ and coupled into a $600 \mu \mathrm{m}$-diameter fibre attached to a spectrometer (Ocean Optics USB2000+ UVVIS). The energy was measured using a pyroelectric sensor (OPHIR, PE9-ES-C). A combination of a quarter wave plate and Glan Thompson polarizer was used to probe the handedness of the circular polarization of the laser emission.

\section{Results and discussion}

Initially, free-standing films that did not contain dye were studied to determine their mechanochromic properties. Films containing different concentrations by weight of the reactive mesogen mixture (RMM) were prepared to allow us to investigate the effect of the polymer density on the mechanical properties of the film in terms of the extent of elongation that could be achieved and the maximum possible spectral shift in the band gap (i.e. the mechanochromic sensitivity, the ratio of the change in the position of the band gap to the strain). Below a concentration of $25 \mathrm{wt} \%$ of the RMM, it was found that the films did not possess adequate structural integrity and thus could not be delaminated from the glass cells without some polymer clearly remaining on either one or both of the glass substrates. Above this concentration, however, the films could be peeled intact without leaving any observable solid polymer residue. At concentrations above $\sim 60 \mathrm{wt} \%$, the films were too rigid to enable substantial elongation before breaking.

The transmission spectra of the different gels were recorded as they were subjected to a uniaxial mechanical strain along a direction perpendicular to the helicoidal axis, which was achieved by increasing the separation of the variable aperture in 10 $\mu \mathrm{m}$ increments. In all cases the initial aperture width was the same $(320 \mu \mathrm{m})$. Upon increasing the aperture width, the films were laterally stretched, leading to a mechanochromic effect whereby the selective reflection band blue-shifted; this is qualitatively consistent with a thinning of the film and a contraction of the pitch. An example of this is shown in Fig. 2a for a mixture with 58 wt $\%$ RMM. Here, the position of the long band-edge (LBE) is found to shift by $63 \mathrm{~nm}$ for a $200 \mu \mathrm{m}$ elongation. Note that the transmission increases on stretching because the opening of the aperture allows more light to pass through to the spectrometer. The PBG is not perfectly sharp due to the presence of the RMM, however it compares favourably to previous work on LC elastomers and the manufacturing process we use here is relatively straightforward. This linear variation is in accordance 


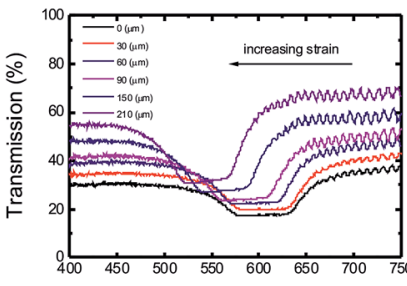

(a)

(c)

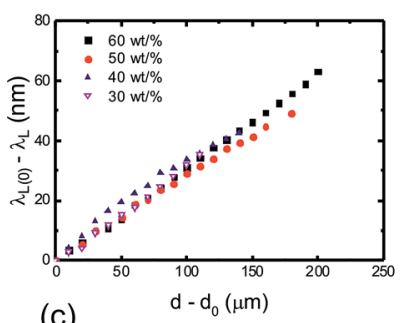

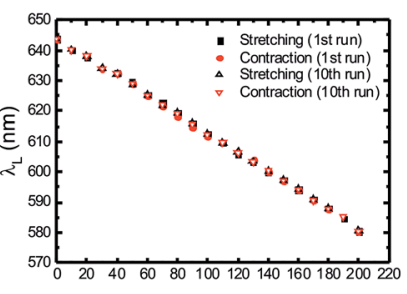

(b)

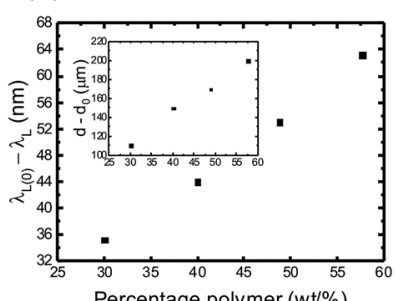

(d)
Fig. 2 Mechanochromic properties of an in situ photopolymerised stretchable gel: (a) the change in transmission spectrum as a $58 \mathrm{wt} / \%$ RMM film is elongated; (b) the change in the long band edge (LBE) position on stretching and contracting; (c) the change in wavelength of the LBE for different concentrations of RMM; (d) the tuning range for different percentages RMM; inset: the maximum elongation before breaking for different percentage polymers ( $x$-axis is the same as the main figure).

with previously reported results, ${ }^{\mathbf{1 6}, 21}$ and is in accordance with a first order approximation of Poisson's ratio. This is further corroborated by qualitative measurements that show that the thickness of the film decreases in a linear fashion as the film is stretched (these results are presented in Fig. S1 of the ESI $\dagger$ ).

Fig. 2b shows the change in the long band-edge wavelength as a function of the relative aperture width for the gel consisting of $58 \mathrm{wt} / \%$ of the reactive mesogen mixture. In this case, measurements were carried out upon both elongation and contraction of the film. Firstly, the results show a linear dependence on the aperture width and, importantly, there is no apparent hysteresis even after more than ten cycles of stretching and relaxing the film. Since there is no hysteresis, it is then possible to select a desired wavelength by controlling the extent of elongation using the variable-width aperture. The lack of hysteresis was not unique to this sample and was also observed in the other gels consisting of lower polymer concentrations for which a structurally intact gel could be formed.

Fig. 2c shows the change in the long band-edge wavelength relative to that of the initial unstrained sample as a function of the aperture width for four gels consisting of different concentrations of the RMM in the same LC host. This data has been obtained from transmission spectra plots of the type shown in Fig. 2a. In all cases, the results show an almost linear decrease in the wavelength upon lateral stretching of the film (perpendicular to the helicoidal axis), with the greatest tuning-range being observed for the $58 \mathrm{wt} \%$ sample: from $\lambda_{\mathrm{L}}-\lambda_{\mathrm{L}(0)}=63 \mathrm{~nm}$ for 58 $\mathrm{wt} \%$ compared to $\lambda_{\mathrm{L}}-\lambda_{\mathrm{L}(0)}=35 \mathrm{~nm}$ for $30 \mathrm{wt} \%$. The LBE for the initial, unstrained films do not perfectly coincide with each other - this is due to slight changes in the pitch of the chiral nematic LC that arises for the different polymer concentrations.
Fig. 2d is a plot of the maximum shift of the long-wavelength edge, $\lambda_{\mathrm{L}(\max )}$, relative to the initial, unstrained wavelength, $\lambda_{\mathrm{L}(0)}$. Also shown in the inset is the relative change in the aperture width as a function of the concentration of polymer before the film breaks. Clearly, the results demonstrate that the larger the concentration by weight of the RMM, the greater the film elongation and therefore the greater the relative tuning range $\lambda_{\mathrm{L}}$ $-\lambda_{\mathrm{L}(0)}$. As the change in the long-wavelength band-edge is $\sim 0.3$ $\mathrm{nm}$ per $\mu \mathrm{m}$ of elongation for each film - regardless of the RMM concentration - it seems that the greater polymer concentration allows elongation over a longer distance, but that a given elongation provides the same degree of tuning. Therefore, the mechanochromic sensitivity is unaltered by the increase in the concentration of the reactive mesogen.

To observe the change in the appearance of the films as they are mechanically stretched, the variable-width aperture was mounted on an optical polarising microscope and images were captured for different aperture separations. Fig. 3a-f provide example images of a film containing $\sim 58 \mathrm{wt} \%$ polymer as it is elongated from 320 to $445 \mu \mathrm{m}$. In the images, the spacer beads $(20 \mu \mathrm{m})$ can be seen, as can some defects in the alignment (e.g. oily streaks). On stretching, the film colour that is observed through crossed polarisers changes from green to orange (a redshift) as the PBG itself blue-shifts and the light reflected by the film changes. Although a colour change can be observed in reflection, it is a subtle change so we used crossed polarisers.

A video of the film being stretched until it breaks can be found in the ESI; $\uparrow$ from this the relaxation time can be seen to be on the order of a second.

Together, these results show that by using a combination of reactive and non-reactive mesogens, a stretchable chiral nematic gel may be created whereby the selective reflection band can be controlled reversibly and selectively - with a given elongation corresponding to a specific wavelength. Our ultimate aim was to demonstrate tuneable laser emission; therefore, the next step was to add a laser dye to one of the gels. To this end, we chose a 58 wt\% RMM concentration, since it showed the greatest degree of tuning; to this we added the fluorescent dye, PM597 (1.0 wt\%, Exciton).

When optically pumped by the second-harmonic output of a 1 ns pulse-width Nd:YAG laser, we observed laser emission
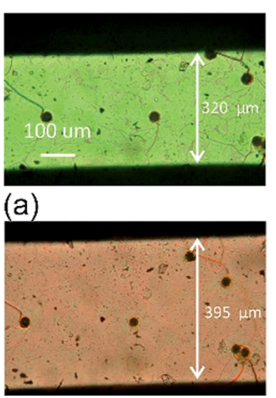

(d)
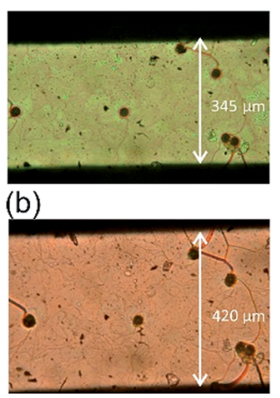

(e)

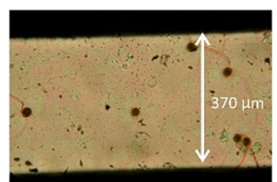

(c)

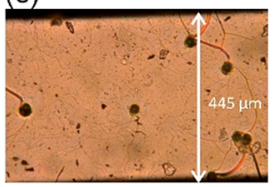

(f)
Fig. 3 Optical polarising microscope images of a 58 wt\% RMM film as it is stretched by: (a) $0 \mu \mathrm{m}$; (b) $25 \mu \mathrm{m}$; (c) $50 \mu \mathrm{m}$; (d) $75 \mu \mathrm{m}$; (e) $100 \mu \mathrm{m}$; (f) $125 \mu \mathrm{m}$. 
from the gel at a wavelength corresponding to the long-band edge. Upon extension of the films, the wavelength of the laser emission was found to blue-shift and was commensurate with the change in the selective reflection band and the decrease in the pitch shown in Fig. $2 b$.

Fig. 4a presents the laser emission spectra for different extensions of the film where it can be seen that the lasing peak shifts to shorter wavelengths as the film is elongated. The full width at half maximum is found to be $\approx 1.7 \mathrm{~nm}$, which is on the same order as the resolution limit of our spectrometer. Fig. $4 \mathrm{~b}$ shows the linear relationship between the laser wavelength and the elongation of the film in accordance with the results obtained for the selective reflection band as seen in Fig. 2. Again, no hysteresis is observed. In this case, the finite bandwidth of the dye's fluorescence spectrum limits the extent over which the laser wavelength can be tuned - and so the largest tuning ranges demonstrated in Fig. 2 are not fully realised here.

Input-output characteristics for the laser gel were obtained for four different film extensions and from these results the excitation laser threshold was obtained from the discontinuity in the differential. The results are presented in Fig. 4c. It is found that the laser threshold exhibits a minimum near $\lambda=582$ $\mathrm{nm}$, which is the gain maximum for PM597 dispersed in E7. This is in accordance with previous studies on LC lasers using PM597 as the laser dye. ${ }^{26}$ Encouragingly, there appears to be no noticeable increase in the excitation threshold after the film preparation process. The results show that the threshold fluences per pulse are approximately the same for the freestanding film when compared to that obtained for the same sample prior to the substrates of the cell being separated so as to delaminate the film.

The results show that as the film is subjected to a uniaxial lateral extension, the laser threshold decreases to a minimum at $582 \mathrm{~nm}$ and then increases as the laser wavelength moves to shorter wavelengths. This is believed to be due to a change in

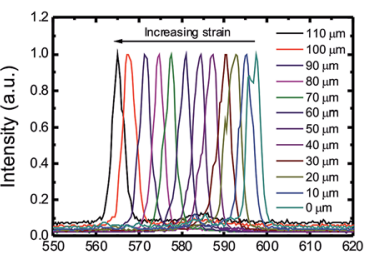

(a) Wavelength $(\mathrm{nm})$

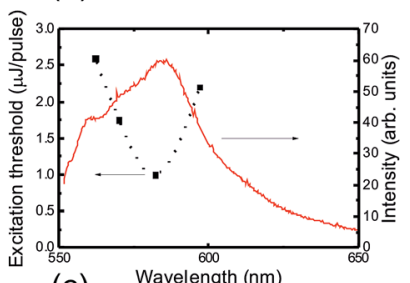

(c)

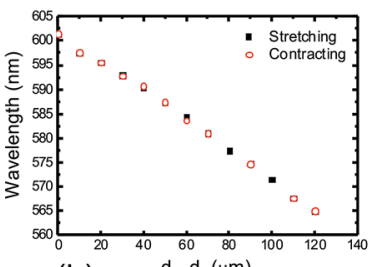

(b)

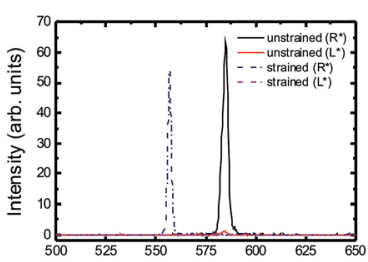

(d)
Fig. 4 A stretchable laser gel: (a) change in laser emission spectrum upon elongation; (b) wavelength of emission on stretching and contracting; (c) change in laser threshold on stretching (black squares, primary axis; the dashed line is to guide the eye) against PM597 gain curve (red line, secondary axis); (d) emission of a stretched and unstretched film for right and left circularly polarised light. the relative position of the band-edge with respect to the gain curve of the laser dye. At wavelengths away from $\lambda=582 \mathrm{~nm}$, the gain curve decreases and there is a concomitant increase in the excitation threshold. Furthermore, the finite extent of the gain curve also explains why the laser tuning range is found to be smaller $(\sim 36 \mathrm{~nm})$ than the shift observed for the selective reflection band in Fig. 2a $(\sim 60 \mathrm{~nm})$. In terms of the tuning range, the limiting factor in this case is actually the gain curve of the laser dye (something further corroborated by the observation that the film could be stretched beyond the point at which laser emission ceased without the film breaking). Thus, a combination of multiple dyes (as shown in earlier studies ${ }^{5}$ ) may allow greater wavelength-tuning ranges to be achieved.

To confirm that the handedness of the polarisation of the laser emission is unchanged during stretching, we have recorded the emission spectrum for both left and right circularly polarised light emitted from the dye-doped chiral nematic LC. Since the helical structure is inherently right handed, we would expect the emission at the long band-edge to be right circularly polarised. Fig. $4 \mathrm{~d}$ shows the circular polarization properties of the dye-doped gel before and after stretching; in both cases, laser emission is observed for right circularly polarized light, which blue-shifts upon stretching, whereas no laser emission is observed for left-circularly polarized light. It is clear that the right-handed helical structure is maintained upon stretching, which is in contrast to previous work on cross-linked elastomers where a change in the polarisation behaviour was observed on stretching. ${ }^{27}$ This is not an unexpected difference given that the materials used here consist of polymer networks containing interstitial LC as opposed to LC molecules that are themselves integral to the polymer structure used in ref. 27 .

Finally, to demonstrate the versatility of these gels and their fabrication process, a multi-coloured film was created by curing half of the device at room temperature and the other half at 45 ${ }^{\circ} \mathrm{C}$. Since the pitch is temperature-dependent, this process locked-in two separate domains with different pitches. Transmission spectra measurements confirmed that the band gap for

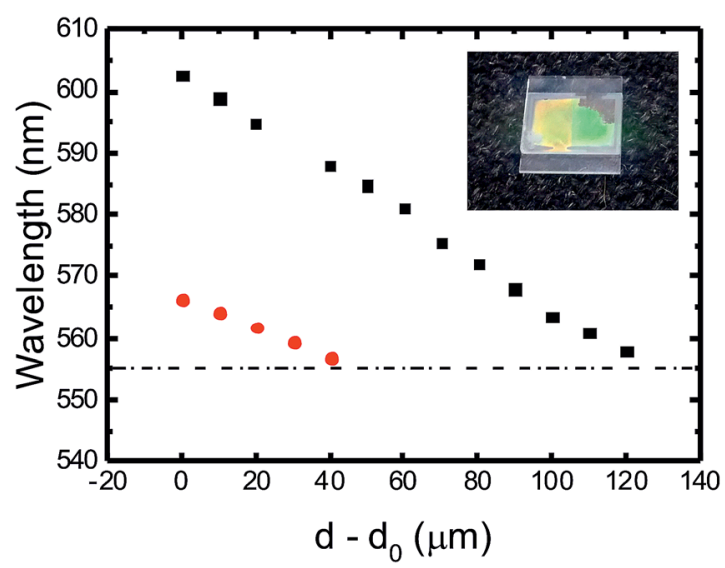

Fig. 5 The change in emission wavelength for two different regions of a patterned gel laser: red dots - short pitch region; black squares long pitch region. The dashed line at $550 \mathrm{~nm}$ shows the limit of the dye gain curve. Inset: a stretchable gel sample exhibiting two regions before delamination. 
each domain occurred at different central wavelength positions. When subjected to a mechanical strain, the band-gaps were found to blue-shift simultaneously, reversibly and without hysteresis. The corresponding laser gel exhibited laser emission at a different laser wavelength (603 and $566 \mathrm{~nm}$ ) from each domain, which was consistent with the different pitch values. On stretching, the laser wavelengths in both regions were found to blue-shift simultaneously. As with the monodomain sample in Fig. 4, the tuning ranges were limited by the gain curve of the dye (lasing was curtailed at $555 \mathrm{~nm}$ for both regions, as shown in Fig. 5); thus, the shorter pitch region exhibited a shorter tuning range ( $\sim 12 \mathrm{~nm}$ compared to a $45 \mathrm{~nm}$ tuning range for the long-pitch sample).

\section{Conclusions}

Here, we have demonstrated a method for creating a stretchable, multi-colored LC laser gel in which the emission wavelength can be precisely selected by controlling the extent of film elongation. To this end, we have presented results on the mechanochromic properties of these LC gels and have shown how the tuning range increases with the concentration of reactive species within the mixture while the mechanochromic sensitivity remains unchanged. By doping these films with fluorescent dyes, we have observed reversible laser tuning across a $40 \mathrm{~nm}$ wavelength range upon stretching and contracting the films. Moreover, the tuning range did not appear to exhibit hysteresis, allowing a desired wavelength to be selected by controlling the elongation of the film. By varying the curing conditions, it was possible to pattern the films to exhibit regions of different pitch with consequently different emission wavelengths. Our simple, versatile manufacturing technique couples with the small dimensions of the lasers and their suitability for solution processing to make them an exciting prospect for a variety of applications such as pressure sensors and microactuators.

\section{Acknowledgements}

SMM gratefully acknowledges the financial support of The Royal Society.

\section{Notes and references}

1 For a recent review see, H. Coles and S. Morris, Nat. Photonics, 2010, 4, 10.

2 J. P. Dowling, M. Scalora, M. J. Bloemer and C. M. Bowden, J. Appl. Phys., 1994, 75, 1896.

3 M. Ozaki, M. Kasano, D. Ganzke, W. Haase and K. Yoshino, Adv. Mater., 2002, 14, 306.

4 W. Cao, A. Muñoz, P. Palffy-Muhoray and B. Taheri, Nat. Mater., 2002, 1, 111.
5 S. M. Morris, P. J. Hands, S. Findeisen-Tandel, R. H. Cole, T. D. Wilkinson and H. J. Coles, Opt. Express, 2008, 16, 18827.

6 H. Xianyu, T.-H. Lin and S.-T. Wu, Appl. Phys. Lett., 2006, 89, 091124.

7 P. J. W. Hands, D. J. Gardiner, S. M. Morris, C. Mowatt, T. D. Wilkinson and H. J. Coles, Appl. Phys. Lett., 2011, 98, 141102.

8 D. J. Gardiner, W.-K. Hsiao, S. M. Morris, P. J. W. Hands, T. D. Wilkinson, I. M. Hutchings and H. J. Coles, Soft Matter, 2012, 8, 9977.

9 J. Schmidtke and W. Stille, Eur. Phys. J. B, 2003, 31, 179.

10 T. K. Mavrogordatos, S. M. Morris, S. M. Wood, H. J. Coles and T. D. Wilkinson, Phys. Rev. E: Stat., Nonlinear, Soft Matter Phys., 2013, 87, 062504.

11 A. Muñoz F, P. Palffy-Muhoray and B. Taheri, Opt. Lett., 2001, 26, 84 .

12 K. Funamoto, M. Ozaki and K. Yoshino, Jpn. J. Appl. Phys., Part 1, 2003, 42, 1523.

13 S. Furumi, S. Yokoyama, A. Otomo and S. Mashiko, Appl. Phys. Lett., 2003, 82, 16.

14 I. Lelidis, G. Barbero and A. M. Scarfone, Cent. Eur. J. Phys., 2012, 10, 587.

15 A. Chanishvili, G. Chilaya, G. Petriashvili, R. Barberi, R. Bartolino, G. Cipparrone, A. Mazzulla and L. Oriol, Appl. Phys. Lett., 2003, 83, 5353.

16 H. Finkelmann, S. T. Kim, A. Muñoz, P. Palffy-Muhoray and B. Taheri, Adv. Mater., 2001, 13, 1069.

17 S. Mayer and R. Zentel, Curr. Opin. Solid State Mater. Sci., 2002, 6, 545.

18 P. Xie and R. Zhang, J. Mater. Chem., 2005, 15, 2529.

19 A. Varanytsia, H. Nagai, K. Urayama and P. Palffy-Muhoray, Sci. Rep., 2015, 5, 17739.

20 S. T. Kim and H. Finkelmann, Macromol. Rapid Commun., 2001, 22, 429.

$21 \mathrm{~J}$. Schmidtke, S. Kniesel and H. Finkelmann, Macromolecules, 2005, 38, 1357.

22 D. J. Broer, H. Finkelmann and K. Kondo, Makromol. Chem. Rapid Comm., 1988, 189, 185.

23 T. K. Mavrogordatos, S. M. Morris, F. Castles, P. J. W. Hands, A. D. Ford, H. J. Coles and T. D. Wilkinson, Phys. Rev. E: Stat., Nonlinear, Soft Matter Phys., 2012, 86, 011705.

24 F. Castles, S. M. Morris, J. M. C. Hung, M. M. Qasim, A. D. Wright, S. Nosheen, S. S. Choi, B. I. Outram, S. J. Elston, C. Burgess, L. Hill, T. D. Wilkinson and H. J. Coles, Nat. Mater., 2014, 13, 817.

25 Y. Inoue, H. Yoshida, K. Inoue, Y. Shiozaki, H. Kubo, A. Fujii and M. Ozaki, Adv. Mater., 2011, 23, 46.

26 C. Mowatt, S. M. Morris, M. H. Song, T. D. Wilkinson, R. H. Friend and H. J. Coles, J. Appl. Phys., 2010, 107, 043101.

27 P. Cicuta, A. R. Tajbakhsh and E. M. Terentjev, Phys. Rev. E: Stat., Nonlinear, Soft Matter Phys., 2002, 65, 051704. 